\title{
Ueber künstliche Trommelfelle aus Paraffin und Silber.
}

Von Privatdozent Dr. B. Gomperz in Wien.

An dieser Stelle hat im Februar d. J. Dr. Hamm (Braun. schweig) mitgeteilt, daß er seit längerer Zeit ein bei $45^{\circ}$ schmelzendes Hartparaffin zu künstlichen Trommelfellen verwende. Seine Methode besteht darin, daß Stückchen sterilisierten Verbandmulls, welche dem Umfang der Trommelfelllücke an Größe entsprechen, in das flüssig gemachte Paraffin eingetaucht und in die Perforation gedrückt werden. Dr. H $\mathrm{amm}$ schreibt, daß. das Paraffin in der Ohrenheilkunde schon mehrfach, aber stets nur nach 'Trepanationen des Warzenfortsatzes zur Abkürzung der Nachbehandlung in Verwendung gezogen wurde, und da seine Darstellung den Eindruck hervorrufen muß, daß die Idee, Paraffin zu künstlichen Trommelfellen zu verwenden, eine neue, und zwar seine eigene sei, kann ich es nicht unterlassen, diesen Irrtum richtig zu stellen.

Ein Gemisch von Vaseline und Paraffin als sehr empfehlenswertes Material zu künstlichen Trommelfellen habe ich nämlich schon 1902 in meinem Vortrage: "Neue künstliche Trommelfelle" gelegentlich der Naturforscherversammlung in Karlsbad empfohlen. Kurze Zeit vorher hatte Gersuny gezeigt, wie ausgezeichnet Vaseline vom lebenden Gewebe vertragen wird, und es lag ja nahe, diese Toleranz auch auf unserem Gebiete zu verwerten. Es war dabei nur notwendig, den Schmelzpunkt so weit zu erhöhen, daß die Festigkeit der Prothese auch bei Fiebertemperaturen nicht gefährdet werde, also auf mindestens $41^{\circ} \mathrm{C}$. Ich will $\mathrm{Dr} . \mathrm{Hamm}$ gewiß nicht vorwerfen, daß ihm die Referate über diesen Vortrag unbekannt geblieben sind, die in den Fachzeitschriften erschienen.') Aber es sind auch ausführlichere Mitteilungen aus meiner Feder über diesen Vortrag verölfentlicht worden, welche schon schwerer

1) Verhandlungen der 74. Versammlung Deutscher Naturforseher und Aerzte 1902, 11, 2. Hälfte, S. 433. Archiv für Ohrenheilkunde Bd. 57, S. 122. Zeitschrift fír Ohrenheilkunde Bd. 43. Monatsschrift für Ohrenheilkunde 1902, S. 444 und 1903, S. 64. 
zu übersehen waren, in den Annales des maladies de l'oreille, Oktober 1902, No. $10^{1}$ ) und in der Wiener medizinischen Wochenschrift 1902, No. 50 und 51.2)

In diesen Arbeiten ist zu lesen, daß ich gelegentlich der Suche nach einem reizlosen Material für künstliche Trommelfelle, die von der Mucosa tympani auf die Dauer vertragen werden sollten, auch auf die Idee kam, Vaseline von hohem Schmelzpunkte zu verwen. den, das ich mir durch Zusammenschmelzen chemisch reinen $\mathrm{Pa}$ raffins mit weißem, säurefreiem Vaseline herstellen ließ. Dieses Produkt hatte einen Schmelzpunkt von $41-42^{\circ}$, und ich kann demnach das Neue, wenn auch nicht das Bessere, in Hamms Empfehlung nur darin sehen, daß er Hartparaffin von $45^{\circ}$ Schmelzpunkt in Verwendung zog; daß der höhere Schmelzpunkt in anbetracht der Empfindlichkeit der Paukenschleimhaut weniger vorteilhaft ist, brauche ich wohl nicht weiter auszuführen. Daß H amm dieses härtere Paraffin in Verbindung mit Verbandmull in die Trommel fellü̈cken einführt, ist meiner Meinung nach weder originell - denn Wattekugeln, die mit Vaselinöl getränkt wurden, stehen seit langem als künstliche Trommelfelle in Gebrauch - , noch empfehlenswert, da wir die Reizlosigkeit des Paraffins durch die Zugabe des Verbandmulls nur verringern.

Es handelt sich bei diesen Prothesen darum, die möglichst größte Reizlosigkeit zu erzielen. Wo bei Vorhandensein von Trommelfelllücken die Auskleidung der Paukenhöhle ihren Charakter als Schleimhaut verloren hat, dadurch, daß sich das Epithel des Gehör ganges oder der Trommelfellkutis in die Paukenhöhle hineingezogen hat, dort werden alle möglichen Arten künstlicher Trommelfelle gut vertragen. Die derbe, sehnig graue, trocken glänzende Membran. welche wir meistens am Grunde der Totalperforationen, ferner bei solchen Lücken beobachten können, welche bis an den Rand des Trommelfells reichen, ferner bei den Defekten, wie sie nach der radikalen Freilegung der Mittelohrräume zurückbleiben, zeigt die größte Toleranz gegenüber den Materialien für künstliche Trommelfelle, die seit langem in Gebrauch stehen.

Es bleibt aber eine große Reihe von Perforationsfällen übrig, bei denen die Mucosa tympani ihren schleimhautartigen Charakter bewahrt hat; diese Fälle vertragen die künstlichen Trommelfelle, die vor meinen neuen in Gebrauch standen, überhaupt nicht, oder auf die Dauer nicht. Weil diese Fälle auch die mit reinem Vaselineöl oder Mentholvaselineöl getränkte Wattekugel - eine der reizlosesten Prothesen - nicht vertrugen, suchte ich die Watte ganz zu eliminieren und Vaseline ohne Träger zu verwenden, und ich muß es direkt als einen Rückschritt betrachten, wenn jetzt $\mathrm{H}$ am für die Paraffinprothesen wieder Verbandmull als Constituens empfiehlt.

Ich habe seinerzeit vorgeschlagen, das Vaseline-Paraffingemisch mittels einer Spritze an die Lücke im Trommelfell zu bringen, und ließ mir dazu eine starke, leicht sterilisierbare Pravazsche Spritze mit Querbügel und Seitenbändern fertigen. Auf den konischen Ansatz sind winklig abgebogene Kanülen aus Feinsilber von $7 \mathrm{~cm}$ Länge und $11 / 2$ bis $2 \mathrm{~mm}$ Durchmesser mit abgerundetem, etwas verdicktem Ende aufzustecken, welche nach Bedarf leicht gekrümmt werden können. ${ }^{3}$ ) Das Vaseline-Paraffingemisch wird flüssig in die Spritze aufgezogen und nach dem Erstarren appliziert; man wärmt dazu die Kanüle nur an ihrem hinteren Ende etwas an und drückt gegen die Lücke gerade so viel von dem Spritzeninhalt los, daB der Defekt bis ins Niveau der Trommelfellflache davon erfüllt wird. Ich habe schon 1902 mitteilen können, daß ich diese Prothese in vielen Fällen mit ausgezeichnet hörverbesserndem Erfolg angewendet und wochen- und monatelang unverändert liegen gesehen habe. Wenn ich diese Vaseline-Paraffinprothesen schon zur Zeit der angeführten Publikationen seltener in Gebrauch zog, so geschah es deshalb, weil ich in chemisch reinem Blattsilber ein Material zu künstlichen Trommelfellen gefunden habe, welches die Forderung nach größter Reizlosigkeit, Dauerhaftigkeit, Schmiegsamkeit, geringem Gewicht, leichter Sterilisierbarbeit und chemischer Beständigkeit in gradezu idealer Weise erfüllte, und weil mir die daraus hergestellten Konvolute bezüglich der funktionellen Wirksamkeit die glänzendsten Resultate ergeben haben, welche sich mit künstlichen Trommelfellen überhaupt erzielen lassen.

Ich habe in den letzten fünf Jahren Fälle genug verzeichnet, wo diese Silberprothesen nicht nur wochenlang, sondern drei, vier, sogar sechs Monate mit unverändert hörverbessernder $\mathrm{Kraft}$ im $\mathrm{Ohr}$ lagen, ohne die geringsten Nebenbeschwerden. Dr. Hamm fand es aber notwendig, um den Wert "seiner" Paraffintrommelfelle besonders hervorzuheben, die Brauchbarkeit meiner Silbertrommelfelle anzuzweifeln. Er zweifelt an ihrer "absoluten" Reizlosigkeit, an dem Anhalten der "absoluten" chemischen Reinheit im Ohr und

1) De l'Amelioration des Troubles de l'Auditioni, suites d'Otite suppurée.

2) Neue künstliche Trommelfelle.

${ }^{3}$ ) Gefertigt von H. Reiner, Wien IX. stellt es in Frage, ob die Silberbleche wohl überall in der nötigen Zurichtung erhältlich sein dürften.

Es sei mir gestattet, auf diese Bedenken zu"antworten. "Ab-

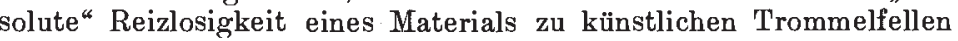
für alle Fälle gibt es nicht, denn die jeweilige Empfindlichkeit der Paukenschleimhaut wird dafür ausschlaggebend sein, ob eine Prothese - auch die beste - auf die Dauer ohne Eiterung vertragen wird oder nicht. Darum spreche ich auch in meiner letzten und ausführlichsten Arbeit') über diesen Gegenstand nur davon, daß dem chemisch reinen Silber unter den vorhandenen Materialien die "größte" Reizlosigkeit eigen sei.

Dr. Hamm meint ferner, die chemische Reinheit der Silbertrommelfelle dürfte nach seinem Erachten im Ohr nicht lange anhalten. Nach meinen Erfahrungen stimmt dies nicht, denn dort, wo das Silbertrommelfell vertragen wird, spielt sich kein chemischer Prozeß ab, der die Reinheit der Silbereinlage irgendwie beeinträchtigen könnte. Wenn man bei solchen Fällen auch nach Monaten die Prothesen auswechselt, so zeigt sich das Silber an der Berührungsstelle blank und weiß. Einer Veränderung der chemischen Reinheit der Silbereinlage, welche durch die Berührung mit der Paukenschleimhaut erfolgen würde, könnte aber kaum eine schädliche Wirkung zugeschrieben werden. V. Urbantschitsch empfahl sogar solche Blattsilbereinlagen als wirksames Heilmittel gegen chronische Paukenhöhleneiterung, die er dadurch öfters zum Stillstand brachte. ${ }^{2}$ )

Was schließlich die Erhältlichkeit der geklopften Silberbleche anbelangt, so gebe ich gern $z u$, daß letztere nicht überall leicht zu haben sind; aber wird z. B. der Zahnarzt einer kleinen Provinzstadt das Zähneplombieren sein lassen, weil an der Stätte seiner Tätigkeit Gold- und Silberfolie schwer oder nicht erhältlich sind?

Uebrigens möchte ich da wieder auf meine angeführten Arbeiten verweisen, in denen Dr. Hamm finden wird, daß es fürderhin nicht notwendig ist, die seinerzeit versuchten, dünngeklopften Silberbleche von 0,02 bis $0,004 \mathrm{~mm} \mathrm{zu}$ beschaffen, da sich mir ,am besten und verwendbarsten Konvolute aus chemisch reinem Blattsilber (Schaumsilber von unter einem Mikromillimeter Dicke) bewährt haben. Dieses Produkt ${ }^{3}$, das zum Echtversilbern vieler Gegenstände gebraucht und im großen erzeugt wird, besitzt neben seiner genügenden Festigkeit und Elastizität die Leichtigkeit eines Spinngewebes, läßt sich ohne die geringste Schwierigkeit einführen, durch sanften Druck adaptieren und durch Ausspritzung wieder entfernen. Von der Weichheit dieser Konvolute erhält man erst eine richtige Vorstellung, wenn man sie zwischen den Fingern zerdrückt. Schon der leichteste Druck verwandelt sie in ein dünnes Plättchen von außerordentlicher, unvergleichbarer Glätte. Hergerichtet werden diese Konvolute derart, daß man je ein quadratisches Blatt von $8 \mathrm{~cm}$ Seitenlänge sechsmal zusammenfaltet, wodurch quadratische Päckchen von 64 Lagen und $1 \mathrm{~cm}$ Seitenlänge entstehen. Ein bis zwei, höchstens drei derart zusammengefaltete Blätter genügen für eine Prothese. Ich lasse mir eine größere Anzahl solcher Konvolute in einem Pulverglas trocken sterilisieren. Dann verwende ich sie im gegebenen Falle nach gründlichster Reinigung des Gehörganges, die ebenso wichtig ist wie die Sterilisierung der Trichter, Tupfer und Pinzetten. Den Gehörgang wische ich bis zum Trommelfellfalz vorsichtig mit $5 \%$ igem Menthol-Vaselineöl aus; von der früher yon mir geübten Reinigung mit Aether-Alkohol sehe ich jetzt meistens ab. Sehr oft tauche ich jetzt die sterilen Silberprothesen vor der Einführung in $5 \%$ iges Menthol-Vaselineől. Dadurch wird die Einführung sehr erleichtert und dem künstlichen Trommelfell ein steriles Lager geschaffen, in dem es monatelang liegen kann. Mit Zuhilfenahme eines dünnen, auf die Sonde gedrehten Wattepinsels, den ich nach meiner Methode sterilisiere, ${ }^{4}$ ) adaptiere ich dann die Silberkonvolute, bis der Defekt bis ans Trommelfellniveau ausgeglichen erscheint.

Ueber die Indikationen habe ich mich in den hier angezogenen Arbeiten, sowie in meinen Publikationen über Trommelfellersatz ${ }^{5}$ und „zur Funktion des Gehörorganes nach der Radikaloperation ${ }^{* 6}$ ) ausgesprochen.

1) Wiener medizinische Wochenschrift 1902 , No. 50 und 51.

2) Oesterreichische otologische Gesellschaft, Sitzung vom 30. Mai 1904. Refer. Internationales Zentralblatt für Ohrenheilkunde, Bd. 3 , No 3 , S. 110.

$\left.{ }^{3}\right)$ „Echtes Blattsilber", verfertigt von C. A. Falk, Wien V, Margarethner Hauptstraße 22, und abgegeben in Büchlein zu 20 Heller à 20 Blatt.

4) Zur Sterilisierung der Tupfer, Pinsel und Einlagen für Ohr und Nase, Zeitschrift f. Ohrenheilkunde Bd. 51. I, S. 87.

5) Drasches Bibliothek der gesamten medizinischen Wissenschaft, Teschen 1899. 6) Wiener medizinische Wochenschrift 1900 , No. 9 u. 10 . 